\title{
Guidelines for the Management of Children and Adolescent with COVID-19: protocol for an update
}

\author{
Qi Zhou ${ }^{1,2 \#}$, Weiguo Li $^{3 \#}$, Siya Zhao ${ }^{4}$, Qinyuan $\mathrm{Li}^{3}$, Qianling Shi ${ }^{1}$, Zijun Wang ${ }^{2}$, Hui Liu ${ }^{4}$, Xiao Liu ${ }^{4}$, Janne \\ Estill $^{5,6}$, Zhengxiu Luo ${ }^{3}$, Qiu Li ${ }^{7}$, Kehu Yang ${ }^{1,2,8,9,10,11,12}$, Enmei Liu ${ }^{3}$, Yaolong Chen ${ }^{2,8,9,10,11,12}$ \\ ${ }^{1}$ The First School of Clinical Medicine, Lanzhou University, Lanzhou, China; ${ }^{2}$ Evidence-based Medicine Center, School of Basic Medical Sciences, \\ Lanzhou University, Lanzhou, China; ${ }^{3}$ Department of Respiratory Medicine, Children's Hospital of Chongqing Medical University, National \\ Clinical Research Center for Child Health and Disorders, Ministry of Education Key Laboratory of Child Development and Disorders, Chongqing \\ Key Laboratory of Pediatrics, Chongqing, China; ${ }^{4}$ School of Public Health, Lanzhou University, Lanzhou, China; ${ }^{5}$ Institute of Global Health, \\ University of Geneva, Geneva, Switzerland; ${ }^{6}$ Institute of Mathematical Statistics and Actuarial Science, University of Bern, Bern, Switzerland; \\ ${ }^{7}$ Department of Nephrology, Children's Hospital of Chongqing Medical University, National Clinical Research Center for Child Health and \\ Disorders, Ministry of Education Key Laboratory of Child Development and Disorders, Chongqing Key Laboratory of Pediatrics, Chongqing, \\ China; ${ }^{8}$ WHO Collaborating Centre for Guideline Implementation and Knowledge Translation, Lanzhou, China; ${ }^{9}$ GIN Asia, Lanzhou, China; \\ ${ }^{10}$ Chinese GRADE Centre, Lanzhou, China; ${ }^{11}$ Lanzhou University, an Affiliate of the Cochrane China Network, Lanzhou, China; ${ }^{12}$ Key Laboratory \\ of Evidence Based Medicine \& Knowledge Translation of Gansu Province, Lanzhou, China \\ \#These authors contributed equally to this work. \\ Correspondence to: Yaolong Chen. Evidence-based Medicine Center, School of Basic Medical Sciences, Lanzhou University, Lanzhou 730000, China. \\ Email: chenyaolong@lzu.edu.cn; Enmei Liu. Department of Respiratory Medicine, Children’s Hospital of Chongqing Medical University, National \\ Clinical Research Center for Child Health and Disorders, Ministry of Education Key Laboratory of Child Development and Disorders, Chongqing \\ Key Laboratory of Pediatrics, Chongqing 400014, China. Email: emliu186@126.com; Kehu Yang. The First School of Clinical Medicine, Evidence- \\ based Medicine Center, School of Basic Medical Sciences, Lanzhou University, Lanzhou 730000, China. Email: kehuyangebm2006@126.com.
}

Background: Coronavirus disease 2019 (COVID-19) has become a constant threat to people's lives, bringing huge challenges to the global public health and medical service system. In order to ensure the timeliness and reliability of the recommendations in guidelines, the working group of the Rapid Advice Guidelines for Management of Children with COVID-19 decided to update the guideline to incorporate the latest evidence to guide the management of COVID-19 in children and adolescent.

Methods: We will update the guidelines, originally developed as a rapid advice guideline, into a standard guideline. We will follow the clinical practice guideline (CPG) update manuals of the National Institute for Health and Clinical Excellence (NICE) and the Spanish National Health System (SNHS). The updated guidelines will also follow the RIGHT (Reporting Items for Practice Guidelines in Healthcare) checklist and Checklist for the Reporting of Updated Guidelines (CheckUp).

Discussion: Through systematic search, evaluation and grading of the best available relevant clinical evidence, combined with the experience of frontline clinical experts in the fight against the epidemic and the wishes of patients and their caretakers, we will update our previous rapid advice guidelines into a highquality, implementable standard guidelines for the management of COVID-19 in children and adolescent.

Trial registration: The standard guideline update has been registered at the International Practice Guidelines Registry Platform (http://guidelines-registry.cn/?lang=en, registration No. IPGRP-2020CN101).

Keywords: Coronavirus disease 2019 (COVID-19); children; standard guideline; protocol

Submitted Sep 12, 2020. Accepted for publication Nov 27, 2020.

doi: $10.21037 /$ tp-20-290

View this article at: http://dx.doi.org/10.21037/tp-20-290

(c) Translational Pediatrics. All rights reserved. 


\section{Introduction}

Rigorous and transparent high-quality clinical practice guidelines (CPGs) provide a solid basis for clinical practitioners for decision-making. CPGs also help to standardize medical practice, as well as improve the quality of medical services, patients' health outcomes and quality of life, and the economic efficiency of healthcare (1-3). However, guidelines that lack the latest evidence may mislead clinical practice and deteriorate the quality of medical services (4). Therefore, guidelines need to be regularly updated to ensure the reliability, timeliness, implementation and clinical relevance of the recommendations $(5,6)$.

Coronavirus disease 2019 (COVID-19) has become a constant threat to people's lives, bringing huge challenges to the global public health and medical service system $(7,8)$. Children as a vulnerable group need special attention in the society $(9,10)$. For this reason, the National Clinical Research Center for Child Health and Disorders developed the first international Rapid Advice Guidelines for Management of Children with COVID-19 following the WHO rapid recommendations guidelines development methodology (11-14). However, in response to the COVID-19 pandemic that is still escalating globally, researchers continue to race against time to find more and better evidence. If the COVID-19 guidelines for children do not incorporate the latest evidence, this will not only lead to a suboptimal use of the latest research findings in clinical practice, but also result in a tremendous waste in health-care resources. Such guidelines would no longer guide health care providers with the best treatment to the children with COVID-19.

In order to ensure the timeliness and reliability of the recommendations in guidelines, the working group of the Rapid Advice Guidelines for Management of Children with COVID-19 decided to update the guideline to incorporate the latest evidence to guide the management of COVID-19 in children and adolescent $(15,16)$. This protocol illustrates the methodology and process for this guideline update.

\section{Methods}

We will update the guidelines, originally developed as a rapid advice guideline, into a standard guideline. We will follow the CPG update manuals of the National Institute for Health and Clinical Excellence (NICE) and the Spanish National Health System (SNHS) $(17,18)$. The updated guidelines will also follow the RIGHT (Reporting Items for Practice Guidelines in Healthcare) checklist and Checklist for the Reporting of Updated Guidelines (CheckUp) $(19,20)$. The standard guideline update has been registered at the International Practice Guidelines Registry Platform (http://guidelines-registry.cn/?lang=en, registration No. IPGRP-2020CN101).

This guideline will not conduct the original research on humans and animals, only the secondary analysis of published articles, so ethical approval is not required.

\section{Guidelines update working group}

The Chair and Co-Chairs of the original Rapid Advice Guidelines for Management of Children with COVID-19 working group will first consult all members whether they would like to participate in the development of the updated guidelines. Those who agree will become members of the guideline update working group. In addition, we will also invite new experts to participate in the updated guidelines development to enhance geographical representativeness. Four specific groups will be established: a steering group, an expert consensus group, an evidence synthesis and evaluation group, and a secretariat.

\section{Steering group}

The group will be established by the National Clinical Research Center for Child Health and Disorders and will aim to include four to six senior clinicians and methodologists from the organization developing the guidelines (a chair, a co-chair, a chief methodologist, and chief clinical experts). The primary responsibilities of the steering group will be to: (I) form the other groups, (II) determine the topic and scope of the guidelines, (III) determine the clinical questions of the guidelines, (IV) monitor and evaluate the guideline updating process, (V) draft the full updated guidelines, and (VI) approve the recommendations and the final version of the guidelines for publication.

\section{Expert consensus group}

The group will comprise 40 to 50 experts from various disciplines, including methodologists, pediatricians, respiratory physicians, infectious disease physicians, general practitioners, nurses, clinical pharmacists, public health specialists, health economists, legal experts, global health researchers, and patient representatives. We will aim at a broad and balanced representation of different disciplines, 
geographical locations, and males and females. The primary responsibilities of the expert consensus group will be to: (I) evaluate the scope of the updated clinical questions, (II) prioritize the new clinical questions suggested to be added to the updated guidelines, (III) participate in the Delphi survey to vote and reach consensus on the recommendations, and (IV) revise the first draft of the full text of the updated guidelines.

\section{Evidence synthesis and evaluation group}

The group will consist of 14 to 16 methodologists and pediatricians. The main responsibilities of the evidence synthesis and evaluation group will be to: (I) retrieve the latest evidence for the ten clinical questions in the original version of the guidelines and form an evidence update report, (II) formulate additional preliminary clinical questions to be included in the update, (III) retrieve, develop or update the systematic evaluation of the evidence for the identified clinical questions, (IV) complete the Grading of Recommendations Assessment, Development and Evaluation (GRADE) evidence grading, and (V) to write the guideline recommendation decision table.

\section{Secretariat}

The secretariat will consist of three to four members. The main responsibilities of the secretariat will be to: (I) organize the meetings related to the guidelines development, (II) collect and manage the conflicts of interest of the guideline working group members, (III) design the scope questionnaire for the new clinical questions and use the questionnaire to assess the research results, and (IV) design the Delphi survey for the recommendations and assessment of research results.

\section{Declaration of interests}

All members will be required to complete a declaration of conflicts of interest prior to participating in the guideline working groups. These declarations will be included as attachments of the final guideline update (21).

\section{Scope of the guidelines}

The target population of the updated guidelines are children and adolescent with severe acute respiratory syndrome coronavirus 2 (SARS-CoV-2) infection (22). The main users of the guidelines are pediatricians, clinical pharmacists, general practitioners and nurses working in general hospitals, children's hospitals, and primary care clinics worldwide. The guidelines focus on the management of COVID-19.

\section{Formulating clinical questions}

The evidence synthesis and evaluation group will formulate clinical questions based on: (I) research gaps identified in our original guidelines, other guidelines and clinical trials for children with COVID-19, (II) external reviewers' feedback, and (III) the ten clinical questions from our original guidelines. The secretariat will propose an initial draft list of clinical questions and send it to the expert consensus group by email. All experts will be asked to rate the questions by their importance on a seven-point scale, ranging from 1 (the question is not important and should not be included in the guidelines) to 7 (the question is extremely important and must be included in the guidelines). Clinical questions achieving high total scores and approved by all steering group members will be included.

\section{Evidence retrieval and synthesis}

\section{Literature selection}

We will conduct systematic searches with the help of information retrieval expert for each question to find the relevant evidence (23). We will search Medline, Embase, the Cochrane Library and three Chinese literature databases (CNKI, CBM and WanFang). We will also search SSRN (https://www.ssrn.com/index.cfm/en/), medRxiv (https:// www.medrxiv.org/) and bioRxiv (https://www.biorxiv. org/) preprint platforms and Google Scholar for studies not indexed in the aforementioned databases. Further potentially relevant studies will be identified from the references of the included articles through a manual review. We will use search terms that aim to find an adequate balance between sensitivity and specificity of the search.

\section{Evidence assessment}

We will use "A Measurement Tool to Assess Systematic Reviews" (AMSTAR) scale $(24,25)$ to evaluate the methodological quality of the included systematic reviews, rapid reviews and meta-analyses. The Cochrane Risk of Bias tool (ROB) (26), the Quality Assessment of Diagnostic Accuracy Studies-2 (QUADAS-2) (27), and the Newcastle Ottawa Scale (NOS) (28) will be applied to evaluate the methodological quality of randomized controlled trial studies, diagnostic accuracy studies and observational 
studies, respectively. Two reviewers will independently screen all retrieved documents. Disagreements will be resolved through discussion with a third reviewer.

GRADE $(29,30)$ will be used to evaluate the quality of systematic reviews. In the GRADE system, the quality of evidence is categorized as high, moderate, low, or very low, using five downgrading factors (risk of bias, imprecision, inconsistency, indirectness, and publication bias) and three upgrading factors (large effect size, dose-response relationship, and plausible residual confounding). An evidence summary table and an Evidence-to-Decision table will be presented in the final guideline document.

\section{Evidence syntheses}

Evidence from high-quality systematic reviews published within one month before the search will be used directly. For high-quality systematic reviews published more than one month ago, evidence synthesis and evaluation group will perform an updated search and evidence synthesis. If only low-quality systematic reviews or no systematic reviews are available, the evidence synthesis and evaluation group will initiate and lead new systematic reviews to synthesize the latest evidence.

\section{Formulation of the recommendations}

The expert consensus group will draft preliminary recommendations based on the Evidence-to-Decision table, after considering the benefits and harms, the patients' values and preferences, equity, feasibility, and acceptability and the health economic aspects. The expert consensus group will further develop the draft recommendations during a tworound Delphi process, and vote on the recommendations. Consensus will be defined as at least $70 \%$ of the panelists agreeing on the recommendation. Each recommendation contained in the guidelines will encompass a direction (in favour or against) and a rating of the degree of strength. The following categories will be used to establish the rating of the strength of a recommendation: strong recommendation means there is confidence that the desirable effects of adherence to a recommendation outweigh the undesirable effects; and conditional recommendation means that the desirable effects of adherence to a recommendation probably outweigh the undesirable effects, but there is not full confidence of that conclusion. All draft recommendations will be submitted to the secretariat for final approval.

\section{Peer review of guidelines}

The guidelines will be drafted by the steering group and submitted to external experts for peer review. The feedback from the reviewers will be collected and discussed thoroughly. Some recommendations or their strengths may be revised if necessary.

\section{Dissemination and implementation of the guidelines}

After the publication, we will use the following methods to disseminate, promote and implement the updated guidelines: (I) the guidelines will be translated into multiple languages, including English, Chinese, Korean and Japanese, (II) the guidelines will be promoted through platforms such as the WHO Collaborating Centre for Guidelines Implementation and Knowledge Translation and Guidelines International Network, (III) the Guideline Working Group will share all the relevant materials related to the guidelines on the website of National Clinical Research Center for Child Health and Disorders, and (IV) we will assist countries and regions to adapt the guidelines considering the differences in health policies and systems, resources, feasibility and equity across the countries.

\section{Discussion}

According to the WHO guidelines development methodology, a standard (instead of rapid) guideline is recommended for public health emergencies that have lasted at least six months. Therefore, although based on a rapid advice guideline, the updated guidelines for the management of COVID-19 in children and adolescent will be developed according to the principles and development process of standard evidencebased guidelines. As the first international update of Rapid Advice Guidelines for Management of Children with COVID-19, the guidelines will focus on the specific problems in the post-epidemic era. Through systematic search, evaluation and grading of the best available relevant clinical evidence, combined with the experience of frontline clinical experts in the fight against the epidemic and the wishes of patients and their caretakers, we will update our previous rapid advice guidelines into a high-quality, implementable standard guidelines for the management of COVID-19 in children and adolescent, with the aim to standardize the medication worldwide, effectively guide clinical evidence-based practice, 
improve patient outcomes and enhance people's ability of assessing treatment options (31).

\section{Acknowledgments}

Funding: This work was supported by grants from National Clinical Research Center for Child Health and Disorders (Children's Hospital of Chongqing Medical University, Chongqing, China) (grant number NCRCCHD-2020EP-01); Special Fund for Key Research and Development Projects in Gansu Province in 2020; The fourth batch of "Special Project of Science and Technology for Emergency Response to COVID-19" of Chongqing Science and Technology Bureau; Special funding for prevention and control of emergency of COVID-19 from Key Laboratory of Evidence Based Medicine and Knowledge Translation of Gansu Province (grant number No. GSEBMKT2020YJ01); The Fundamental Research Funds for the Central Universities (lzujbky-2020-sp14); The National Key R\&D Program of China (grant number 2018YFC1705500); Research on Response to the Coronavirus Disease 2019 Outbreak in Gansu Province (grant number 20ZD016).

\section{Footnote}

Conflicts of Interest: All authors have completed the ICMJE uniform disclosure form (available at http://dx.doi. org/10.21037/tp-20-290). The authors have no conflicts of interest to declare.

Ethical Statement: The authors are accountable for all aspects of the work in ensuring that questions related to the accuracy or integrity of any part of the work are appropriately investigated and resolved. This guideline will not conduct the original research on humans and animals, only the secondary analysis of published articles, so ethical approval is not required.

Open Access Statement: This is an Open Access article distributed in accordance with the Creative Commons Attribution-NonCommercial-NoDerivs 4.0 International License (CC BY-NC-ND 4.0), which permits the noncommercial replication and distribution of the article with the strict proviso that no changes or edits are made and the original work is properly cited (including links to both the formal publication through the relevant DOI and the license). See: https://creativecommons.org/licenses/by-nc-nd/4.0/.

\section{References}

1. Institute of Medicine (US) Committee on Standards for Developing Trustworthy Clinical Practice Guidelines. Clinical Practice Guidelines We Can Trust. Washington (DC): National Academies Press (US); 2011. Available online: https://www.ncbi.nlm.nih.gov/books/NBK209532/

2. Chen $\mathrm{Y}$, Wang $\mathrm{C}$, Shang $\mathrm{H}$, et al. Clinical practice guidelines in China. BMJ 2018;360:j5158.

3. Yang K, Chen Y, Li Y, et al. Editorial: can China master the guideline challenge? Health Res Policy Syst 2013;11:1.

4. Woolf SH, Grol R, Hutchinson A, et al. Clinical guidelines: potential benefits limitations and harms of clinical guidelines. BMJ 1999;318:527-30.

5. Laine C, Taichman DB, Mulrow C. Trustworthy clinical guidelines. Ann Intern Med 2011;154:774-5.

6. Qaseem A, Forland F, Macbeth F, et al. Guidelines International Network: toward international standards for clinical practice guidelines. Ann Intern Med 2012;156:525-31.

7. Phelan AL, Katz R, Gostin LO. The Novel Coronavirus Originating in Wuhan, China: Challenges for Global Health Governance. JAMA 2020;323:709-10.

8. Li Q, Guan X, Wu P, et al. Early transmission dynamics in Wuhan, China, of novel coronavirus-infected pneumonia. N Engl J Med 2020;382:1199-207.

9. Clark H, Coll-Seck AM, Banerjee A, et al. A future for the world's children? A WHO-UNICEF-Lancet Commission. Lancet 2020;395:605-58.

10. Clark H, Coll-Seck AM, Banerjee A, et al. After COVID-19, a future for the world's children? Lancet 2020;396:298-300.

11. Norris SL. Meeting public health needs in emergenciesWorld Health Organization guidelines. J Evid Based Med 2018;11:133-5.

12. Schünemann HJ, Hill SR, Kakad M, et al. Transparent development of the WHO rapid advice guidelines. PLoS Med 2007;4:e119.

13. Liu E, Smyth RL, Luo Z, et al. Rapid advice guidelines for management of children with COVID-19. Ann Transl Med 2020;8:617.

14. Li W, Zhou Q, Tang Y, et al. Protocol for the development of a rapid advice guidelines for management of children with SARS-CoV-2 infection. Ann Palliat Med 2020;9:2251-5.

15. Vernooij RW, Sanabria AJ, Solà I, et al. Guidance for updating clinical practice guidelines: a systematic review of 
methodological handbooks. Implement Sci 2014;9:3.

16. Shekelle P, Eccles MP, Grimshaw JM, et al. When should clinical guidelines be updated? BMJ 2001;323:155-7.

17. National Institute for Health and Care Excellence. The guidelines manual. Available online: https://www.nice. org.uk/process/pmg6/resources/the-guidelines-manualpdf-2007970804933

18. Working Group for CPG Updates. Updating Clinical Practice Guidelines in the National Health System: Methodology Handbook. National Health System Quality Plan of the Spanish Ministry of Health and Social Policy. Aragon Health Sciences Institute (I+CS), 2009. Clinical Practice Guidelines in the National Health System: I+CS No.2007/02-01. Available online: https://portal.guiasalud. es/wp-content/uploads/2019/06/manual_actualizacion_ gpc_ingles-2.pdf

19. Chen Y, Yang K, Marušić A, et al. A reporting tool for practice guidelines in health care: the RIGHT statement. Ann Intern Med 2017;166:128-32.

20. Vernooij RW, Alonso-Coello P, Brouwers M, et al. Reporting items for updated clinical guidelines: checklist for the reporting of updated guidelines (CheckUp). PLoS Med 2017;14:e1002207.

21. Wang X, Chen Y, Yao L, et al. Reporting of declarations and conflicts of interest in WHO guidelines can be further improved. J Clin Epidemiol 2018;98:1-8.

22. The United Nations International Children's Emergency Fund (UNICEF). Convention on the Rights of the Child. Available online: https://digitalcommons.ilr.cornell.edu/ cgi/viewcontent.cgi? article $=1007 \&$ context $=$ child

23. Li L, Tian J, Tian H, et al. Network meta-analyses could be improved by searching more sources and by involving a

Cite this article as: Zhou Q, Li W, Zhao S, Li Q, Shi Q, Wang Z, Liu H, Liu X, Estill J, Luo Z, Li Q, Yang K, Liu E, Chen Y. Guidelines for the Management of Children and Adolescent with COVID-19: protocol for an update. Transl Pediatr 2021;10(1):177-182. doi: 10.21037/tp-20-290 librarian. J Clin Epidemiol 2014;67:1001-7.

24. Shea BJ, Grimshaw JM, Wells GA, et al. Development of AMSTAR: a measurement tool to assess the methodological quality of systematic reviews. BMC Med Res Methodol 2007;7:10-7.

25. Pieper D, Buechter RB, Li L, et al. Systematic review found AMSTAR, but not R(evised)-AMSTAR, to have good measurement properties. J Clin Epidemiol 2015;68:574-83.

26. Higgins JP, Altman DG, Gøtzsche PC, et al. The Cochrane collaboration's tool for assessing risk of bias in randomised trials. BMJ 2011;343:d5928.

27. Whiting PF, Rutjes AW, Westwood ME, et al. QUADAS-2: a revised tool for the quality assessment of diagnostic accuracy studies. Ann Intern Med 2011;155:529-36.

28. Wells GA, Shea BJ, O'Connell D, et al. The NewcastleOttawa scale (NOS) for assessing the quality of nonrandomized studies in meta-analyses [S/OL]. [202001-19]. Available online: http://www.ohri.ca/programs/ clinical_epidemiology/nosgen.pdf

29. Guyatt GH, Oxman AD, Vist GE, et al. GRADE: an emerging consensus on rating quality of evidence and strength of recommendations. BMJ 2008;336:924-6.

30. Norris SL, Meerpohl JJ, Akl EA, et al. The skills and experience of GRADE methodologists can be assessed with a simple tool. J Clin Epidemiol 2016;79:150-8.e1.

31. Wang Q, Austvoll-Dahlgren A, Zhang J, et al. Evaluating people's ability to assess treatment claims: Validating a test in Mandarin from Claim Evaluation Tools database. J Evid Based Med 2019;12:140-6. 